\title{
Beam-Plasma Discharge in Space and in a Lab
}

\author{
E. G. Shustin* \\ Fryazino Branch, Kotelnikov Institute of Radio Engineering and Electronics, Russian Academy of Sciences, \\ Fryazino, Russia \\ *e-mail:shustin@ms.ire.rssi.ru
}

Received January 22, 2021; revised February 5, 2021; accepted February 5, 2021

\begin{abstract}
The process and applications of a specific type of gaseous discharge-beam-plasma discharge (BPD) - are reviewed. A brief survey of the BPD theory is presented. The basic features of BPD in active geophysical experiments with injection of electron beams into Earth's ionosphere are discussed. Studies of the physics of BPD have revealed the effects successively applied in plasma technology for processing nanoelectronic materials and structures.
\end{abstract}

Keywords: gas discharges, active geophysical experiments, plasma technologies, plasma-processing reactors, thin films, graphene

DOI: $10.1134 / \mathrm{S} 1063780 \mathrm{X} 21060143$

\section{INTRODUCTION: HISTORICAL REVIEW}

This review is devoted to the history and successful applications of a phenomenon of plasma physics: beam-plasma discharge (BPD). It is shown here that this effect has found application in a wide area of human activity and is very useful in such different fields as active geophysical experiments and technologies for creating solid-state electronic devices. Of course, this description is colored by the author's personal experience and does not pretend to be complete: there are reviews on some specific manifestations of BPD referenced in this text. The author believes that this phenomenon will have even wider application in nanotechnologies.

The prehistory of a BPD begins with theoretical description of the phenomenon that was found out in 1949 independently in theoretical works by A.I. Akhiezer and Y.B. Fainberg [1] and D. Bohm and E.P. Gross [2]: when an electron beam moves through a plasma, instability develops, which manifests as increasing thermal fluctuations in the velocity and density of both electrons and the beam at a frequency near plasma Langmuir frequency. The first experimental confirmation of this effect was described in [3].

Let us recall the main points of beam instability theory [4-6].

Below we use the following notation:

$T_{e}$ and $n_{\mathrm{p}}$ are the plasma electron temperature and density; $\omega_{\mathrm{p}}=\left(e^{2} n_{\mathrm{p}} / m \varepsilon_{0}\right)^{1 / 2}$ is the electron plasma frequency, $e$ and $m$ are the charge and the mass of an electron, $M$ is the mass of ion; $c$ is the light velocity; $V_{0}$, $V_{\perp}$ are longitudinal and transverse velocities of beam electrons; $B$ is magnetic field induction, $p$ is the oper- ation gas pressure, and $k$ is a wave number $\Gamma$ is an increment of the instability; the subscript " $b$ " refers to quantities corresponding to the electron beam.

In the simplest model, the plasma is a collisionless, cold $\left(T_{e}=0\right)$, and isotropic continuum with a dielectric constant $\varepsilon_{\mathrm{p}}=1-\omega_{\mathrm{p}}^{2} / \omega^{2}$. For the case of an infinite electron beam with density $n_{\mathrm{b}}$ moving at constant velocity $V_{0}$ through the plasma, it is easy to obtain the dispersion equation from the Poisson equation and equations of motion and continuity:

$$
\frac{\omega_{\mathrm{p}}^{2}}{\omega^{2}}+\frac{\omega_{\mathrm{pb}}^{2}}{\left(\omega-k V_{0}\right)^{2}}=1 \text {. }
$$

The physical mechanism of instability is determined by reversal of the sign of the Coulomb forces between the electron bunching: for $\omega<\omega_{\mathrm{p}} \varepsilon_{\mathrm{p}}<0$, and the charges induced by the beam in the plasma are phased in such a way that the electrostatic forces of the space charge are not repelled a bunch of electrons in the beam, but are directed inside the bunch and compact it.

The instability is very strong: at comparable geometric dimensions and beam parameters the amplification coefficient of the oscillations is greater by an order of magnitude than the gain in a traveling wave tube. This, as well as the lack of complex structural elements (slow-wave structures), immediately attracted the attention of experts in microwave electronics [7].

Equation (1) is obtained under the assumption that all electrons of the beam have the same initial (unperturbed) velocity. In reality, there is always a spread of 
velocities, which is determined by the origin of the beam and can be small relatively to mean velocity (a beam is created in a laboratory plasma using an electron gun) or significantly exceed the thermal spread, for example, when it is generated in a gas discharge plasma or in outer space (flares of third type in the Sun [8], so-called named "bump on tail"). These situations qualitatively differ both in increments of instability and in the nature of nonlinear processes during its development.

Nonlinearity of the interaction is expressed in such phenomena as the deviation of the law of increase in the amplitude of oscillations from exponential; deformation of the beam electron distribution function on energies; changing of the spectrum of oscillations (generation of harmonics and combination frequencies).

Here we will not go into detail about the beam instability theory, redirecting the reader to the monographs cited above $[5,6]$. We only note that, as shown in $[9,10]$, the quasilinear beam instability theory [11, 12] is inapplicable for describing nonlinear processes in a beam-plasma system with a small velocity spread of the beam in (hydrodynamic beam instability): this system should be considered a stochastic self-oscillating system with internal feedback, described by dynamic chaos theory [13].

The formation of BPD, i.e. the creation of a plasma with a high concentration (significantly higher than that expected from collisions of beam electrons with gas molecules), when the beam propagated through a rarefied gas in a strong longitudinal magnetic field, was discovered and explained qualitatively in [14-16] already at an early stage of experimental studies of beam-plasma instabilities. There the ignition thresholds of the BPD in a high magnetic field, the oscillations spectra and the types of instabilities responsible for the formation and maintenance of the BPD, the energy relations in the BPD were experimentally studied.

The main manifestations of BPD in a high magnetic field $\left(B^{2} / 8 \pi \gg n_{\mathrm{p}} T_{e}\right)$ are: an abrupt increase in plasma density at a certain (threshold) value of the beam current, a significant increase in the diameter of the plasma column compared to the diameter of the electron beam, a clear high-frequency gain radiation from the plasma region and the transformation of its spectrum (from monochromatic or set of harmonics to a broadband spectrum in the $\omega_{\mathrm{p}}$ band), a sharp increase in low frequency fluctuations.

In $[17,18]$ was shown that BPD can develop without an external magnetic field. Features of optical radiation from the region of BPD without an external magnetic field were described in [17]. Four stages of the BPD formation were observed at the gas pressure increasing (see Fig. 1): strictly outlined area of the region occupied by the beam, scattered by collisions of beam electrons with neutral particles (electron beam

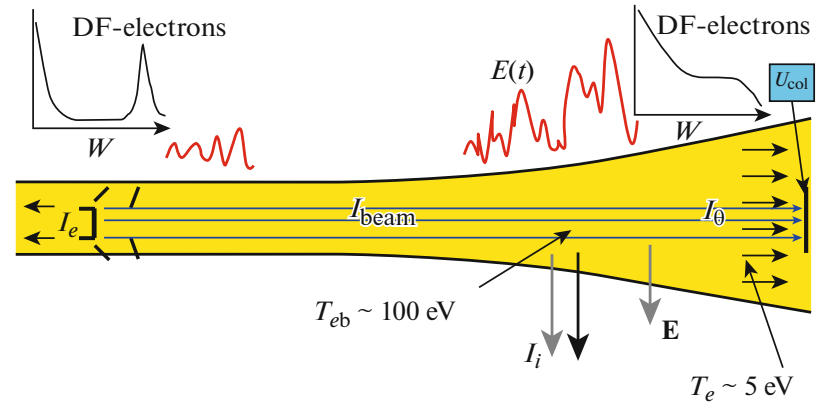

Fig. 1. Scheme of the formation of BPD.

excited plasma, EBEP), thin shining channel at regime of the beam ion focusing, BPD configuration in shape of shining ellipsoid connected with the beam injector by thin channel of EBEP and the track of the beam scattered in the high density gas.

The area occupied by BPD is distinctly more than the volume of the beam, if $B$ is not too much (Larmour radius of electrons $R_{\mathrm{L} e}=m V_{0} / e B \ll R_{0} \ll R_{\mathrm{L} i}=$ $\left.M V_{0} / e B\right)$.

The first works on BPD theory appeared in 1976 $[19,20]$ and were stimulated mainly by willingness to apply the phenomenon in microwave electronics [14], and later by the desire to explain and describe the features observed in active geophysical experiments and in laboratory experiments simulating their conditions $[21,22]$.

\section{PHYSICS OF BPD}

Summing up the results of theoretical, experimental, and computer simulation studies, we can construct the following qualitative pattern of excitation and properties of BPD (Fig. 1).

At the initial stage of the discharge (after injection of the electron beam into a gas medium begins), the gas is ionized due to collisions of the electron beam with molecules. At a small beam density or low gas pressure, if the ionization rate exceeds the rate of escape of charged particles from the ionization region, this "primary" plasma is accumulated and creates an EBEP. At sufficient densities of the beam current and the gas, when a certain density of primary plasma accumulates in the system, beam instability begins to develop. For a given set of experimental parameters, the ignition of the discharge occurs when the beam current exceeds a certain threshold value, which is associated with these parameters by the empirical dependence [23]

$$
I_{\mathrm{t}}=\frac{E_{0}^{3 / 2}}{B_{0} L} f(p)
$$

where $f(p)$ is a function with a minimum and varies about as $p^{ \pm \beta}(0.5<\beta<1)$ up and down this minimal 


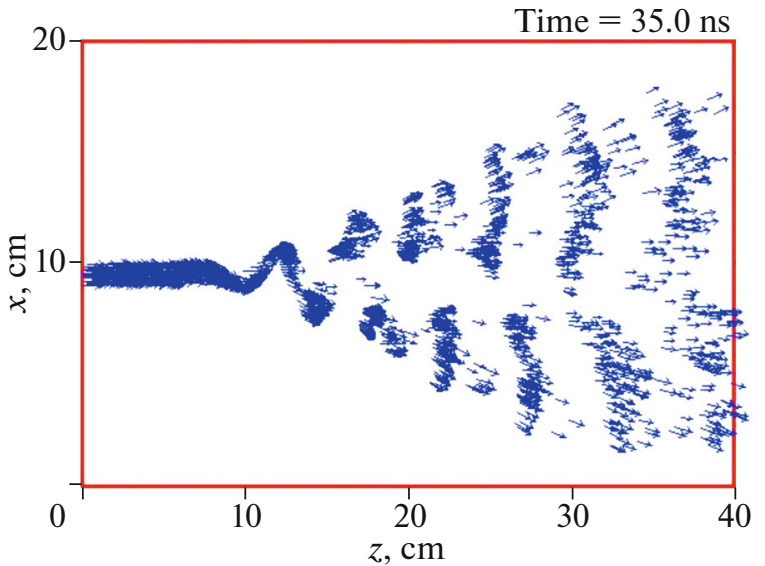

Fig. 2. Velocity vectors of the beam electrons in the BPD.

pressure. For example, for $E_{0}=(1-3) \mathrm{keV}, B \sim 40 \mathrm{G}$, $L \sim 10 \mathrm{~m}, p_{\min }=(1-2) \times 10^{-5}$ Torr. $^{1}$

The ionization rate of neutral atoms during BPD ignition increases 2 order of magnitude compared to the rate of collisional ionization by the beam under the same conditions. Since the rate of diffusion also increases when plasma temperature increases, the density of the discharge plasma increases by a smaller value.

The generation of regular oscillations changes rather quickly, after some beam transit times, by excitation of broadband stochastic oscillations; this is a consequence of oscillation accumulation in the bounded plasma volume and of permanently changing initial conditions for the beam entering the volume $[24,25]$. A highly nonequilibrium plasma with mean energy of electrons up to hundreds of electronvolts is formed in the area occupied by the beam, thus distribution functions of electrons of the beam and of the plasma become physically indiscernible. Electrons from this area create an additional current to the end plates of the chamber. An increase of the electron current results in the growth of plasma potential in the area occupied by the beam.

Energy exchange between an electron beam and plasma during a BPD in a closed cavity excited by the electron beam was analyzed in [26]. Qualitative characteristics of energy exchange (such as beam energy losses and the energy distributions of beam electrons and plasma particles leaving the discharge) both during spontaneous discharge excitation and in the presence of initial beam modulation by regular or noise-like signals are determined. The total losses of the beam power in the discharge vary randomly in time. Their mean value reached $\sim 40 \%$ in the conditions of this computer experiment, and the fraction of

\footnotetext{
${ }^{1}$ Under space experiments conditions, the BPD ignition threshold is determined by the beam removal due to movement of the rocket across the magnetic field.
}

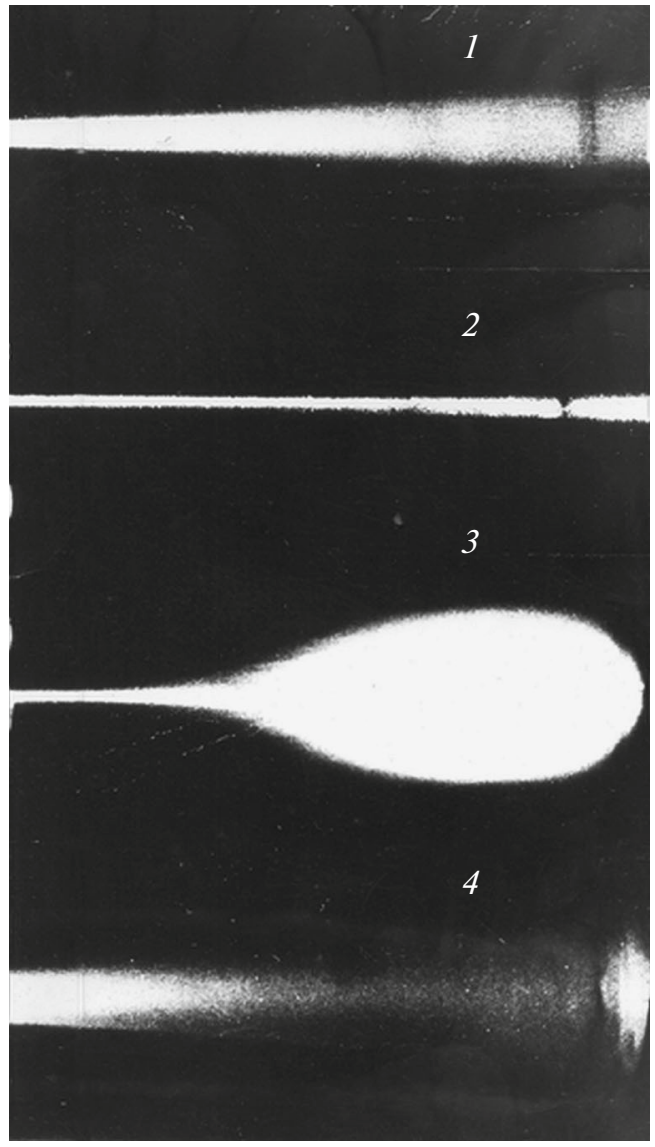

Fig. 3. View of the region of beam propagation in the plasma chamber. The working gas is helium. $p=(1) 0.06$; (2) 0.25 ; (3) 0.7 ; and (4) $7 \mathrm{MPa}$.

the beam power spent on the generation of the ion flow to the periphery of the discharge was $1.5 \%$.

The nonlinear development of instability leads to rapid scattering of electrons both in energy, up to $150 \%$, and in angle $[25,27,28]$ (prompt electron echo) (a clear illustration of this process can be seen in Fig. 2-the result of computer simulation of the beam-plasma system without a magnetic field [27], and Fig. 3 is a photo of the BPD region without a magnetic field at various pressures of the working gas [17]). The diffusion of the beam electrons in terms of velocities creates a final plateau-like distribution of electrons in the velocity space, heating of the plasma electrons and a corresponding increase in the ionization rate by the plasma electrons. In this case, the acceleration of a noticeable part of the beam electrons is observed.

Thus creation of secondary plasma with density essentially excelling the one of the primary plasma takes place. This plasma is turbulent: due to intensive oscillations its effective temperature exceeds essentially the temperature of the primary plasma. Temperature in the intense ionization region is as high as 
$100 \mathrm{eV}$ by an order of magnitude. At the same time, a significant group of superheated electrons with energies up to $\mathrm{I} 50 \mathrm{eV}$ is formed (about $20 \%$ of the total plasma density). ${ }^{2}$

An important property of BPD in the case when it is ignited in a dielectric chamber is noted in [29]. The plasma density and the electron temperature of the discharge are 1.2-3 times greater than for the plasma formed by electron beam transport in a metal vacuum chamber. An additional inflow of energy into the discharge is created with secondary electrons emitted from the cavity surface by beam electrons and with plasma ions accelerated in the near-wall sheath. The authors noticed that this property may result in efficient ion etching of the inner surface of dielectric vessels, which effect can be useful for technology applications and, from the other side, can be the source of contamination in the operation volume.

\section{APPLICATIONS}

Starting from 1972-1974, experimental BPD studies in laboratory conditions pursued mainly three goals: to study the prospects of its application in microwave electronics and in plasma chemistry and also to simulate the conditions of rocket and satellite experiments with electron beams injection into nearearth space.

Experimental studies of BPD began in several laboratories that time in order to study the prospects for its application in plasma chemistry. This idea attracted the attention of researchers in connection with the problems of nonequilibrium plasma chemistry: since the temperatures of ions and electrons in a BPD differ significantly even at the highest pressures of the working medium [30]. Thus, chemical reactions and the production of materials are possible that are not possible in conventional equilibrium chemistry. With the aim of using BPD in plasma chemistry, the kinetics of plasma particles in SPR was mainly studied, as well as the conditions for the excitation of SPR at a relatively high gas density [30, 31]. This application of BPD did not become widespread, primarily due to the low productivity of such processes (the maximum working pressure of the medium in which the discharge was ignited was several $\mathrm{mPa}$ ).

In the 1960s, plasma generators of a noise signal were developed at the Institute of Radio Engineering and Electronics of the USSR Academy of Sciences and at the Kharkov Institute of Physics and Technology for use in electronic warfare devices. However they could not compete with created in the same years vacuum generators of noise based on circuits with traveling wave tubes (TWT) [13]: their efficiency was significantly lower than in TWT, they were poorly controlled, although the frequency tuning capabilities

\footnotetext{
${ }^{2}$ K. I. Gringauz, E. V. Mishin, N. M. Shutte, and A. S. Volokitin, Adv. Space Res. 1, pp. 69-76 (1981).
}

were higher than that of TWT. Applications in microwave electronics have been implemented in the form of microwave devices in the form of hybrid devices, in which metal slow-wave structures filled by plasma were applied. This allowed to increase the beam current and consequently the power of the devices and to provide alteration of their frequency band. Further this direction led to the birth of relativistic plasma electronics [32].

Quickly it became clear that the use of BPD as a plasma source for microwave devices is unacceptable: this plasma is extremely unstable, since it depends on the power of the generated microwave oscillations. Therefore, relativistic devices use an independent plasma source with a tubular electron beam with energy of several hundred $\mathrm{eV}$ (so-called electron beam excited plasma-EBEP). Nevertheless, we present here for information the main parameters of such devices [32]. To date, plasma generators and amplifiers have been created in the $2.4-3.1 \mathrm{GHz}$ band with a power of 100-150 MW, electronic frequency alteration in the $30 \%$ band, efficiency of $10-15 \%$, and pulse duration of 200-300 ns. Compared to devices of vacuum relativistic electronics, these devices, although they have a lower limiting power, but gain in pulse duration and the possibility of rapid (electronic) tuning.

In the next sections we will discuss the history and current state of researches and applications of BPD in space and near-space science and in technologies for the synthesis and processing of materials for nanoelectronics.

\section{BPD IN SPACE}

Researches of effects of electron beams injection from probe rockets into the upper atmosphere were stimulated by the works on tracing the Earth magnetic field and by intention to create artificial aurora (AA). Auroras occur when fluxes of energetic electrons precipitate along the magnetic field into the upper atmosphere at altitudes below $\sim 130 \mathrm{~km}$. Data from in situ measurements of some properties could not be explained solely by collisional degradation of energetic electrons, but demands collisionless beam-plasma interactions to be taken into account. It was thus straightforward intention to employ electron beams injected from a space vehicle with controlled parameters to explore AA in the upper atmosphere Such active experiments on AA with controlled parameters have been conducted using probe rockets and Spacelab. AA experiments commenced January 26, 1969 [33], when an Aerobee 350 rocket was fired from Wallops Island, Virginia, carrying an electron accelerator. Above $230 \mathrm{~km}$, the electron guns put out a beam of $0.5 \mathrm{~A}$ of $10 \mathrm{keV}$ electrons in pulses of 1-s duration aimed downward along the earth's magnetic field lines. The interaction of electron beam with the atmosphere at an altitude of about 100 kilometers generated 
the aurora rays, which could be photographed on the ground by television camera systems.

When an electron beam is emitted in near-Earth space, the problem arises of charging the apparatus and the corresponding deceleration or termination of beam emission. Therefore, the first experiments used an ion or plasma source for charge compensation. However, it was quickly discovered that, at least at altitudes of 100-120 km, the beam creates plasma dense enough for such compensation due to BPD excitation.

Later, experiments were carried out on the French-Soviet program Zarnitsa and ARAKS [3436], which aimed to create an AA. Electron beam and cesium plasma injectors were launched by meteorological rockets onto a ballistic trajectory with an apogee of $155 \mathrm{~km}$. Figure 4 shows the near-missile glow and beams of artificial radiance in the Zarnitsa 2 experiment [37]. It also shows the vertical profile of the leftmost ray with the superimposed profile calculated by the Monte Carlo method. Below $120 \mathrm{~km}$, the PS brightness is consistent with the calculations, but above this level, the observed luminosity significantly exceeded the level determined by collisions of the beam with the gas. In addition, the lower peak is somewhat wider than the profile of natural PSs, which indicates some additional spread in the beam energy $(\sim 10 \%)$.

Similar results were obtained in the Polar 5 experiment [38, 39], carried out in February 1976. The electron accelerator on the "daughter" detachable apparatus emitted a pulsed electron beam with a maximum current of $0.13 \mathrm{~A}$ and an energy of $\sim 10 \mathrm{keV}$. The "mother" load was carried by a $391.4 \mathrm{~nm}$ photometer (the wavelength corresponding to a photon emitted during the act of nitrogen ionization) and diagnostic devices for monitoring scattered and secondary electrons, as well as wave effects. The payloads were separated slowly, so their distance across the Earth's magnetic field reached by the end of the flight, after $80 \mathrm{~min}$.

The observed illumination level follows a neutral density below $130 \mathrm{~km}$, but practically remains constant in the altitude range from $150 \mathrm{~km}$ to an apogee of $220 \mathrm{~km}$ and is much higher than that determined by collisions of the beam with neutrals.

Thus, the observed near-rocket glow, suprathermal electrons, VHF radio emission and the fine altitudinal structure of AA rays point to much stronger interaction of injected electrons with the upper atmosphere than provided by electron collisions. The observations of artificial auroral rays far beneath the rocket indicate that the energy density of plasma waves and concomitant energization of plasma electrons are enhanced in a narrow layer termed the plasma turbulence layer (PTL) [40, 41].

The PTL formation results in a double-peak structure of artificial auroral rays (Fig. 4). Some examples of optical and radar observations of natural auroral
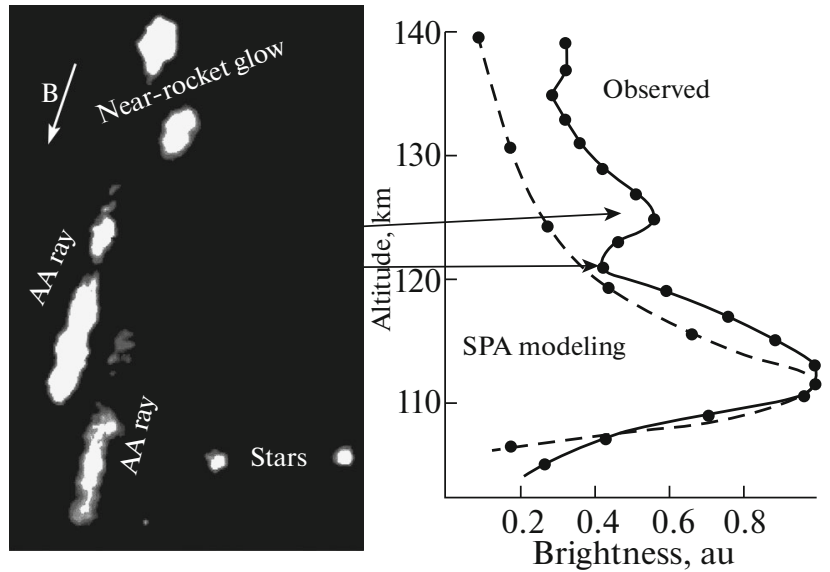

Fig. 4. An example of near-missile glow and artificial radiance rays in "Zarnitsa 2." On the right is the vertical profile of the left: ray of AA with the superimposed Monte Carlo profile (from [53]).

arcs also exhibit two peaks of the luminosity and ionization profiles (so called "enhanced aurora"). An evident resemblance between enhanced and artificial aurora points to their common generation mechanism [40].

The interesting results on topic of beam instability in active experiments are represented in [42]. The experiment was carried out in 1992 onboard the Intercosmos-25 satellite and the Magion-3 daughter satellite (the APEX experiment). The main results are excitation of HF and VLF-LF waves and the generation of fast charged particle flows, which were recorded on both satellites. A satisfactory agreement has been achieved between the calculated parameters of the BPD excited during the injection of a warm electron beam into the cold ionospheric plasma and the HF fields measured at the Magion-3 subsatellite.

The latest information about the experiment with artificial aurora can be found in [44]. Here it says: "...the 12.1-meter-long ICI-5 sounding rocket was launched in Ny-Ålesund, Svalbard, Norway, Nov. 26, 2019." In the message [44], the video shows glowing clouds against the background of a natural aurora observed when a rocket was launched, although the message from Andoya said nothing about the injection of artificial electron beams into the ionosphere. I have not found yet any scientific information on this event.

Apparently, the first works on laboratory modeling of effects arising in active geophysical experiments with electron beams were $[17,18,45]$. These were stimulated by the start of the active preparation of the geophysical Zarnitsa experiment. Then, it was suggested that a BPD can be created in active experiments with injection of electron beams into the Earth's ionosphere and that the BPD may be the cause of the most intense forms of auroras. The Zarnitsa and ARAKS experiments confirmed these hypotheses, 
and later the main researches of the PRD were developed mainly in relation to problems of active geophysical experiments.

Among experiments simulating the conditions of ignition of PRD in the upper atmosphere, we indicate primarily a series of studies carried out by groups from various scientific organizations in the NASA Johnson Space Center (JSC), Houston, USA [46, 47]. They used a test vacuum chamber, which comprises the vessel with diameter of $16.8 \mathrm{~m}$ and a height of $27.4 \mathrm{~m}$, pumped to a residual pressure of $10^{-6}$ Torr. The camera is equipped with means for creating a constant magnetic field of up to $2 \mathrm{~Hz}$, a background plasma with $n_{\mathrm{p}} \sim 10^{6} \mathrm{~cm}^{3}$, and a temperature of up to several electronvolts. Experiments were carried out on this installation to determine the ignition conditions of the $\mathrm{BPD}$, the energy distributions of the beam in the BPD, and other BPD properties and parameters. The laboratory studies on simulating active geophysical experiments with electron beams were also performed in France, Norway, and Russia [48-50]. In [50], in particular, a "flickering" BPD mode was discovered: spontaneous extinction and ignition of a discharge with a frequency on the order of $1 \mathrm{~s}$, caused by burnout of the discharge: imbalance of charged particles due to accelerated diffusion of ions at a relatively low gas density. We also note an interesting effect discovered in the Georgia-60-Spurt space experiment [51] and simulated in [52]: an anomaly in the charging of an electron-emitting apparatus: in a certain range of altitudes, the apparatus's potential rose to a value $35 \%$ higher than the accelerating voltage of the beam. It was shown that this effect is explained by the finite time of flight of electrons returning to the spacecraft when the gas density is insufficient to ignite the BPD.

A detailed review of research concerning BPD in application to natural and artificial auroras can be found in [53]; therefore, we do not continue this topic here.

\section{APPLICATIONS IN PLASMA TECHNOLOGIES}

Beam plasma in plasma processing reactors for microelectronic materials technologies was first applied in [54] and began to be used intensely in late 1990s (see, e.g., [55, 56]). It has witnessed the most diverse applications in studies by S.G. Walton's team at Naval Research Laboratory, USA (see [57-59] and references therein).

A low-density $\left(1-10 \mathrm{~mA} / \mathrm{cm}^{2}\right)$ beam is used; thus, a "calm" plasma is formed by collisional ionization by beam electrons with gas molecules. There are no intrinsic ion or plasma acceleration mechanisms in this system. The low $T_{e}(\sim 1 \mathrm{eV})$ results in production of a flux of ions with low kinetic energy $(<5 \mathrm{eV})$ at adjacent surfaces. The ion kinetic energy can be elevated using the substrate biasing technique, the distribution

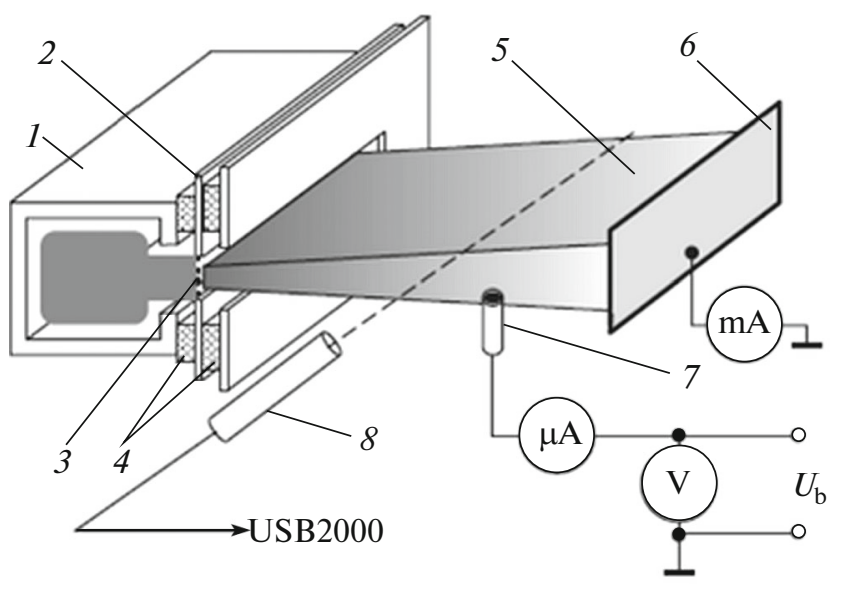

Fig. 5. Beam-plasma reactor with a plasma cathode [60]. (1) Hollow ribbon cathode; (2) anode; (3) extractor; (4) insulating plates; (5) electron beam; (6) collector;

(7) probe; and (8) spectrometer.

is relatively narrow. The electron beam current provides a straightforward "knob" to tune plasma density. Simple changes in neutral gas density or gas composition provide an additional control over the flux intensity and chemistry. However, a relatively high pressure of $1-100 \mathrm{mPa}$ is needed to obtain sufficient ionization; thus the ion flow is influenced by collisions and the directivity of the ion flow is too low.

A similar installation is described in [60-64]. Figure 5 shows a general diagram of installations of [5764]. Their electrical parameters are the same: $U_{\mathrm{b}}=1-$ $3 \mathrm{kV}, I_{\mathrm{b}}=100-500 \mathrm{~mA}$ (current density $1-5 \mathrm{~mA} / \mathrm{cm}^{2}$ ). Also the same are parameters of plasma: $n_{e} \sim 10^{16} \mathrm{~m}^{-3}$, $T_{e}=1-2.5 \mathrm{eV}$. However the second set operates at essentially more pressure $1-10 \mathrm{~Pa}$, so this plasma is highly collisional. This set is used for modification of surfaces of ceramics and glasses and for sintering composites, so it is not an installation for nanoelectronics. A description of the technological applications of installations of the type described in [60] can be found in [61-64] and other works of the Tomsk team led by E.M. Oks.

In Russia, studies on the applications of BPD for plasma processing technologies for micro- and nanoelectronics started in the late 1990s. Then, we began to study the ion component properties during BPD. Figure 6 shows a diagram of the experimental installation and an image.

We revealed that BPD in a low gas pressure can be a source of ion flow with energies of 10-100 eV [65]. These ions propagate from the core of the discharge to its periphery normally to the discharge axis. Their energies substantially exceed the electron thermal energy and can exceed the energy acquired by the ions in the electrostatic field between the discharge plasma column and the chamber wall. 
(a)

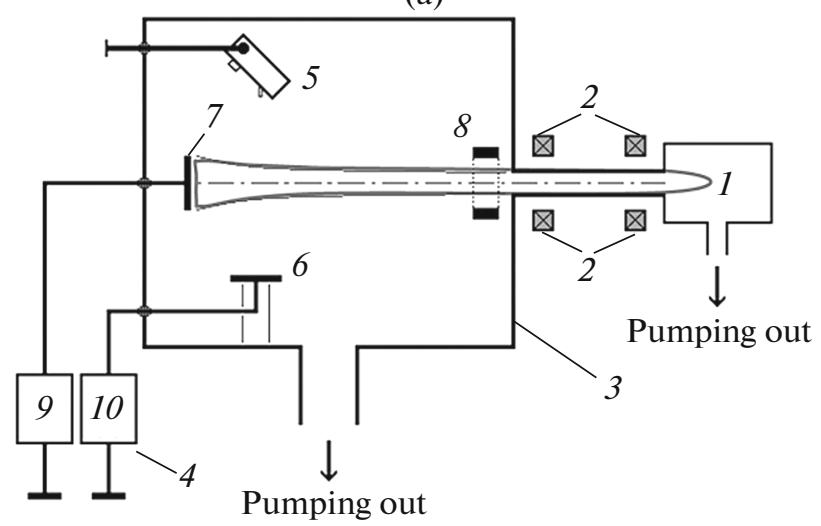

(b)

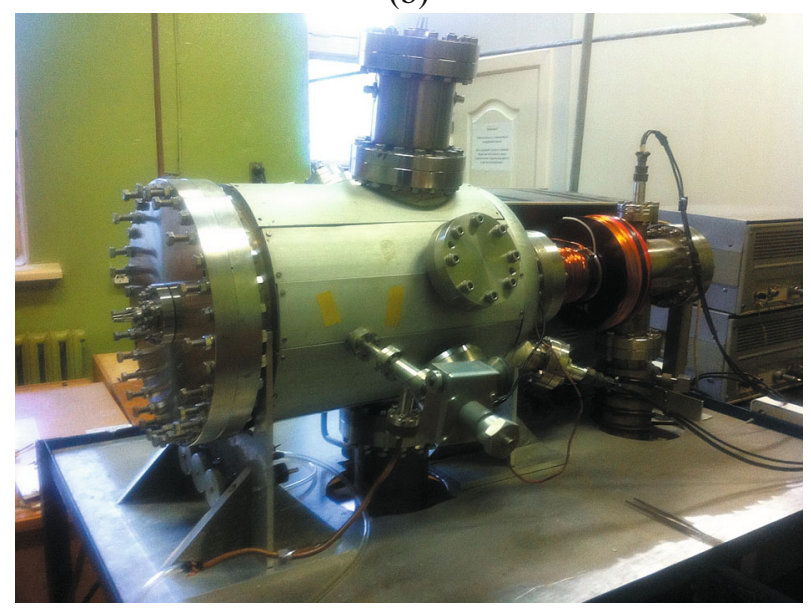

Fig. 6. (a) Scheme and (b) photo of the BPD reactor. (1) electron injector; (2) focusing coils; (3) vacuum chamber; (4) power supplies for controlling the potentials of the collector and substrate; (5) ion energy analyzer; (6) substrate holder; (7) discharge collector; $(8)$ modulating ring, and $(9)$ and (10) power supplies.

Convenient methods for controlling the distribution function of ions acting on a conducting structure on an insulating substrate comprise in varying the high-frequency sinusoidal voltage bias on the substrate electrode. This way results in a typically broad, often bimodal form of the ion distribution function on energies (IDF). As it is well-known, the shape of the IDF curve on the substrate surface significantly affects chemical reactions on a processed surface.

For BPD-based plasma reactor (BPDR) two methods were proposed for controlling the ion distribution function [66-68]. In the first case, a periodic pulsed voltage was applied to the substrate holder; in the second case, the pulsed voltage was applied to the beam collector, thus modulating the plasma potential. We tested these techniques experimentally and by computer simulation to investigate the spatial distributions of the BPD plasma density and the ion flux energy to the substrate holder of plasma processing reactor. The
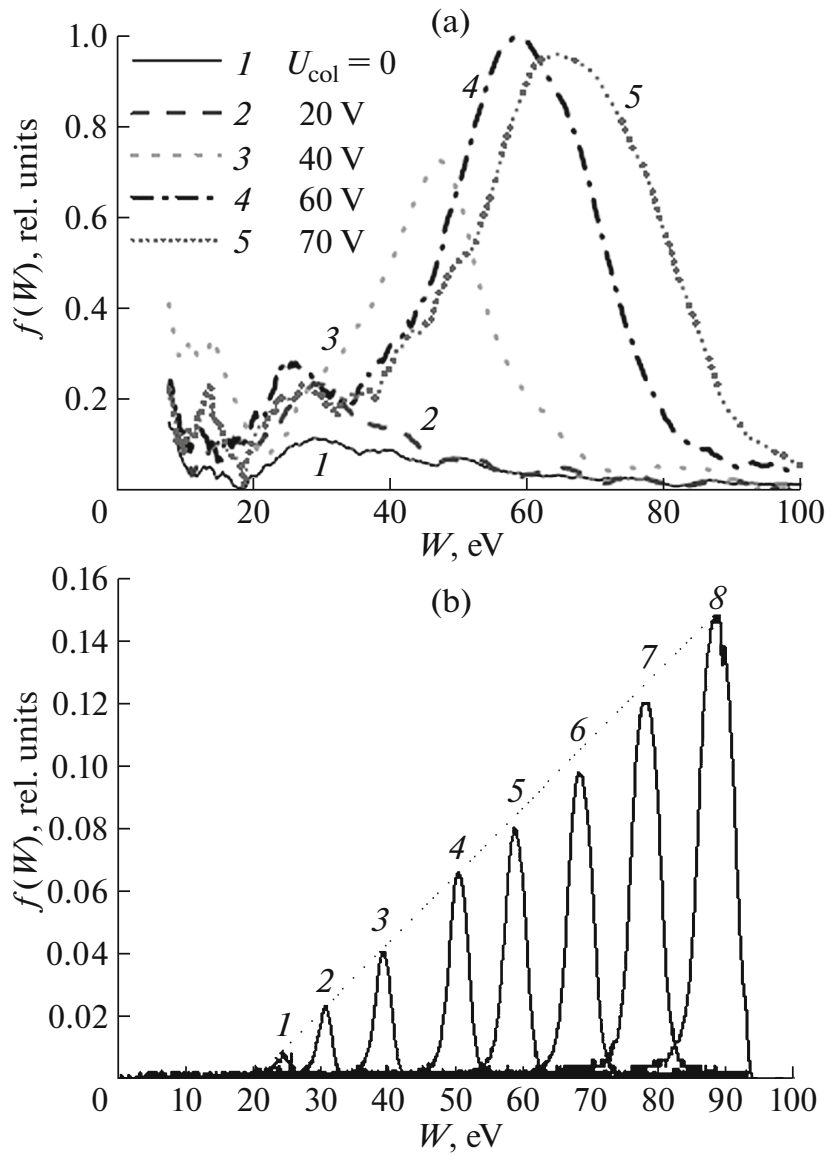

Fig. 7. Distribution of the energy of ions acting on the grounded electrode at the side wall of the plasma chamber at different voltages of the discharge collector: (a) $U_{\mathrm{b}}=$ $2 \mathrm{kV} ; I_{\mathrm{b}}=350 \mathrm{~mA} ; H_{0}=2 \mathrm{mT}$; (b) the same with a discharge at $I_{\mathrm{b}}=150 \mathrm{~mA}$.

comparison showed that the second method provided a more efficient control of the distribution function of the ions, acting on the treated substrate (Fig. 7). In addition, it makes it possible to simultaneously process samples on several substrates at different energies of the ions acting on them, which makes this method effective in scientific research. Thus, a novel plasma processing reactor for treatment of materials used in electronics engineering was designed and created (Fig. 6b) [69].

Changing the external parameters of a BPD in the interaction chamber and using velocity controlling electron beams, the energy of the ions acting on the surface of a sample placed near the side wall of the chamber can be varied within 10-70 eV (Fig. 7). That ion energy range is characteristic for plasma-processing reactors for surface treatment of materials for semiconductor electronics and acousto-electronics, such as etching and deposition of thin films. 


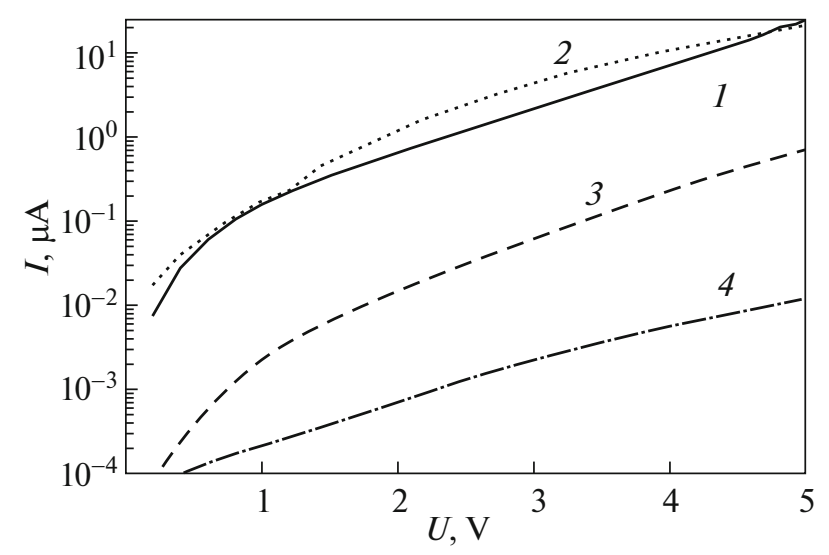

Fig. 8. Current-voltage characteristics of DLC film samples deposited at different pulse voltages on the modulating electrode.

\subsection{Application for Etching the Surface of Microstructures}

The first application of a BPDR was the technology of soft etching of AlGaAs barrier layers in semiconducting $\mathrm{AlGaAs} / \mathrm{InGaAs} / \mathrm{GaAs}$ heterostructures, exploitable for design of microwave field transistors $[66,67]$. Etched samples were analyzed with an atomic force microscope (see Fig. 8) and by measuring the concentration and mobility of a two-dimensional electron gas sensitive to radiation defects.

The rate of etching of GaAs was $3 \mathrm{~nm} / \mathrm{min}$ at ion mean energy $60 \mathrm{eV}$. Because low energy etching is required mainly for preparation of gate grooves of field-effect transistors, and the etching depth should not exceed $10-20 \mathrm{~nm}$, the rate obtained is quite sufficient for industrial application.

We proposed and tested the BPD reactor for fabrication of monoatomic graphite layers (graphene) [70]. Thin graphite crystals of thickness from ten to hundreds of atomic layers with lateral sizes about $0.5 \mathrm{~mm}$, workpieces for subsequent etching, were obtained by splitting natural graphite single crystals using adhesive tape, which was then dissolved in acetone. Such a method made it possible to obtain thinner initial graphite crystals. The produced thin graphite crystal flake was transferred to a polycor substrate and subjected to ion etching.

Indium electrodes were attached to sample boundaries, and the crystal was thinned by plasma etching in the BPD reactor in an argon atmosphere. The film thickness was measuring in situ by the resistance at the plasma etching. The resistance time behavior made it possible to estimate the etch rate and to determine its final time point: the resistance of a square of a homogeneous monolayer is estimated as $\sim 2-3 \mathrm{k} \Omega$ depending on shape of the sample. Argon ion energy was $50 \mathrm{eV}$ at the initial etching stage and $20 \mathrm{eV}$ at final stage, thus minimizing the probability of introducing radiation defects.

Graphite films fabricated with such a way were characterized by Raman spectroscopy. Thus, for the first time, samples of structurally perfect two-layer graphene were manufactured whose characteristic size exceeded $100 \times 100 \mu^{2}$ and several samples of multilayered graphene (FLG) with characteristic size above $500 \times 500 \mu \mathrm{m}^{2}$.

Thus, the new technique of fabricating atomic thin films from natural layered materials with relatively large lateral sizes was demonstrated and proved its usefulness.

\subsection{Carbon Films Deposition}

We applied our reactor for deposition of carbon films by PECVD techniques [71]. For this aim we modified its scheme and regimes: the discharge collector was made from graphite and fed with a voltage of the cathode. The plasma potential was controlled with the additional electrode, which was a short tube, set near the chamber inlet coaxially to the electron beam. Thus carbon atoms and ions were produced by sputtering the collector material, and energy of ions $C$ and Ar was controlled by the way of varying the modulating electrode voltage. This technique differs from usual schemes of PECVD synthesis of DLC films by the way of cracking hydrocarbon gases in gas discharge, providing more simple control of energy of an ion flow and also more uniform composition of atoms and ions acting to the subtract. The samples of nanosized DLC films thus produced at various energies of ions acting onto deposited film had different electrophysical properties depending on this energy. The films with the maximal resistance and the breakdown voltage were obtained at mean ion energy $100 \mathrm{eV}$ (see Fig. 8).

The method of deep level charge relaxation spectroscopy (DLTS) revealed the effect of adsorbed water vapor and alcohol on the electrical properties of the films, which indicates the possibility of using the obtained films as an active adsorbing material for chemical sensors [71].

\subsection{Patterning of Microstructures}

Now interest has been growing in developing nanoelectronic devices including "microwire on isolator" types of structure. For example, patterning graphene into a nanoribbon can open a bandgap that can be tuned by changing the ribbon width, imparting semiconducting properties.

The successful application of the technology for producing defect-free nanoscale films with in situ control of their thickness stimulated the proposal to obtain in this way two-dimensional structures that are promising for use in nanoelectronics and spintronics. 
(a)

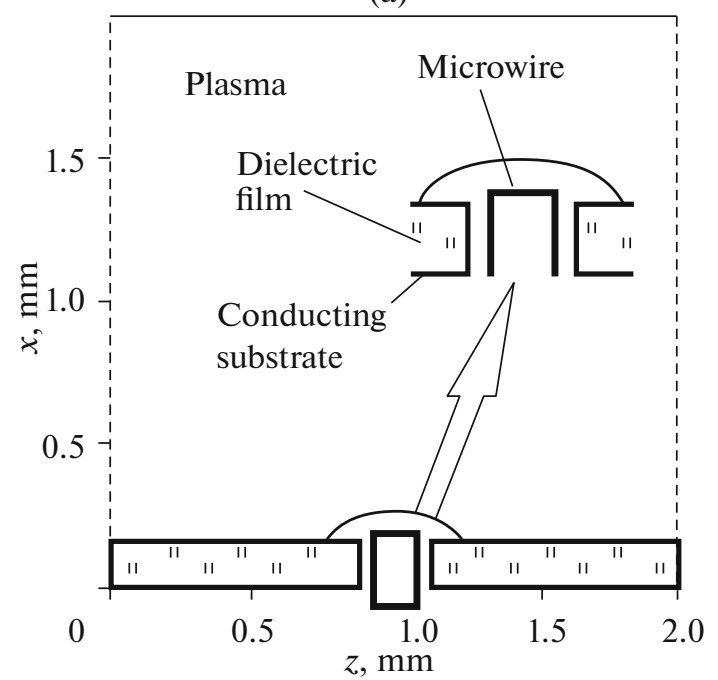

(b)

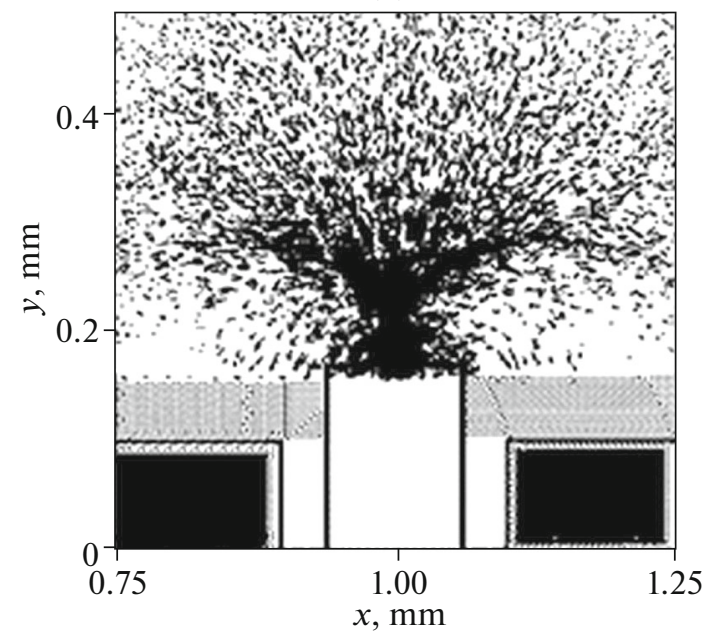

Fig. 9. (a) Geometry of the simulated system and (b) distribution of argon ions in the $(X, Y)$ plane at a constant electrode potential of $70 \mathrm{~V}$.

Thus, work was started on obtaining nanoribbons of a topological insulator by etching pristine single crystals of this material, similar to the method by which samples of two-layer graphene were obtained. These experiments yielded a mixed result: the desired etching effect was observed for ions with energies $\geq 70 \mathrm{eV}$ acting on the initial ribbons, but the resulting structures were found to be highly heterogeneous in thickness. (Because of this relatively disappointing result, information about these experiments was not published.) There was a natural assumption that the unevenness was caused by the inhomogeneity of the ion flow, and it may be possible to exclude or weaken the negative charging effect on the dielectric by means of pulse modulation of the conductor potential. Thus, we started computer modeling of the etching process when conductors on a dielectric substrate are subject to plasma treatment. Simulation was performed by the KARAT code [72, 73].

We investigated the action of ion flows from plasma onto the surface of a microwire, which is a flat conductor, lying on an insulator, with width less than plasma Debye length. The aim of that work was to study the effects of charging a dielectric surface in a complex configuration consisting of a microwire on an insulating substrate. The main qualitative effect of charging was clearly shown in [74]: the formation of an electrostatic lens, leading to a substantial inhomogeneity in the profile of the ion beam acting on the microwire (see Fig. 9).

Then the model was constructed allowing computer simulations of the near-wall area of a planar plasma sheet in conditions where the steady state of the plasma is supported by the production of charged particles in a region removed from the wall [75]. Calculations revealed variation in the energy distribution of the electrons in both time and spatially over the sheet width (cooling the electronic component) due to absorption of fast electrons at the walls bounding the plasma volume. It is shown that the plasma density profile across the sheet width has an abrupt decrease at the boundary of the region of plasma regulation. Let us recall that the standard concept of the potential and plasma density distributions in the sheath and presheath is based on the assumption of a stable energy distribution for the electrons in the presheath [76]. Thus it was shown, that this concept yields inaccurate results for the plasma sheath where the ionization source is remote from the wall.

At last, a model has been designed to allow investigation of dynamics of the plasma adjacent to the microwire, either with a steady state potential applied to the microwire, or under the action of periodic pulses [77]. It was revealed that more complex pulse shapes than rectangle one can provide a very much more homogeneous distribution of the etching rate (at the $10 \%$ level) over the microwire surface. The use of a quasi-noise signal as a control voltage is of greatest interest in this regard. Study of such modes is currently carried out by methods of computer, physical and technological experiments.

\section{CONCLUSIONS}

Plasma processes are actively being developed for nanoelectronic applications. Multiple research groups are involved in the study, and industrial equipment for nanoelectronic technologies is available. Evidently, the corresponding problems can be solved using both improvement of the known processes and schemes and application of qualitatively new approaches. Some of them employ new sources of electron, ion and neutral beams both for the creation plasmas with specific properties and for etching structures in the absence or decrease of surface charge and structural damages 
caused by UV and particle radiation. New materials, in particular, ones of A3B5 and A2B6 groups, carbon nanotubes, graphene, organic semiconductors, and biosupermolecules are actively studied and employed in nanoelectronics. This circumstance results in the widespread application of atomic-layer processes with ultimately low concentrations of defects and accurate control of reacting components and their energies. Many problems of processing A3B5 and A2B6 materials are solved using purely chemical processes, without the use of plasma, but these processes are inapplicable when processing carbon materials. We believe that the processes with pulsed electron beams for plasmas creation are promising tools for the atomic-layer defectfree etching and deposition of atomic-layer films.

\section{ACKNOWLEDGMENTS}

The author is deeply grateful to his colleagues and collaborators in this work: N.V. Isaev ${ }^{\dagger}$, D.V. Kolodko, V.A. Luzanov, E.N. Mirgorodskaya, I.A. Sorokin, V.P. Tarakanov, and M.P. Temiryazeva for valuable participation in the research, which served as the foundation of this review. The author is also grateful to the reviewer of this article, whose comments made it possible to supplement and clarify its content.

\section{FUNDING}

The study was carried out under a state task and partially was supported by the Russian Foundation for Basic Research (project nos. 18-29-19047, 19-07-00432).

\section{OPEN ACCESS}

This article is licensed under a Creative Commons Attribution 4.0 International License, which permits use, sharing, adaptation, distribution and reproduction in any medium or format, as long as you give appropriate credit to the original author(s) and the source, provide a link to the Creative Commons license, and indicate if changes were made. Theimages or other third party material in this article are included in thearticle's Creative Commons license, unless indicated otherwise in a credit line to the material. If material is not included in the article's Creative Commons license and your intended use is not permitted by statutory regulation or exceeds the permitted use, you will need to obtain permission directly from the copyright holder. To view a copy of this license, visit http://creativecommons.org/licenses/by/4.0/.

\section{REFERENCES}

1. A. I. Akhiezer and Ya. B. Fainberg, Dokl. Akad. Nauk SSSR 69, 555 (1949).

2. D. Bohm and E. P. Gross, Phys. Rev. 75, 1851 (1949).

\footnotetext{
${ }^{\dagger}$ Deceased.
}

3. I. F. Kharchenko, Ya. B. Fainberg, R. M. Nikolaev, E. A. Kornilov, E. A. Lutsenko, and N. S. Pedenko, Sov. Phys.-JETP 11, 493 (1960).

4. Ya. B. Fainberg, Sov. At. Energy 11, 958 (1962).

5. R. J. Briggs, Electron-Stream Interaction with Plasmas (MIT Press, Cambridge, MA, 1964).

6. A. B. Mikhailovskii, Theory of Plasma Instabilities (Atomizdat, Moscow, 1971; Consultants Bureau, New York, 1974).

7. G. A. Bernashevskii, E. B. Bogdanov, V. Ya. Kislov, and Z. S. Chernov, Plasma and Electronic Microwave Oscillators and Amplifiers (Sov. Radio, Moscow, 1965) [in Russian].

8. V. V. Zheleznyakov, Radio Emission of the Sun and Planets (Nauka, Moscow, 1964; Pergamon, New York, 1970).

9. V. A. Lavrovskii, I. F. Kharchenko, and E. G. Shustin, Sov. Phys.-JETP 38, 1117 (1973).

10. L. Yu. Kochmarev, A. I. Chmil', and E. G. Shustin, Plasma Phys. Rep. 21, 241 (1995).

11. A. A. Vedenov, E. P. Velikhov, and R. Z. Sagdeev, Sov. Phys.-Usp. 4, 332 (1961).

12. V. D. Shapiro and V. D. Shevchenko, Sov. Phys.-JETP 15, 1053 (1962).

13. N. N. Zalogin and V. Ya. Kislov, Broadband Chaotic Signals in Radio Engineering and Information Systems (Radiotekhnika, Moscow, 2006) [in Russian].

14. I. F. Kharchenko, Ya. B. Fainberg, R. M. Nikolaev, E. A. Kornilov, E. I. Lutsenko, and N. S. Pedenko, Sov. Phys.-Tech. Phys. 6, 551 (1962).

15. L. D. Smullin and W. D. Getty, Phys. Rev. Lett. 9, 3 (1962). https://doi.org/10.1103/PhysRevLett.9.3

16. W. D. Getty and L. D. Smullin, J. Appl. Phys. 34, 3421 (1963). https://doi.org/10.1063/1.1729220

17. E. G. Shustin, V. P. Popovich, and I. F. Kharchenko, Radiotekh. Elektron. 18, 649 (1973).

18. V. P. Popovich, T. A. Novskova, I. F. Kharchenko, and E. G. Shustin, Izv. Vyssh. Uchebn. Zaved., Radiofiz. 16, 1109 (1973).

19. A. A. Galeev, E. V. Mishin, R. Z. Sagdeev, V. D. Shapiro, and V. I. Shevchenko, Sov. Phys.-Dokl. 21, 641 (1976).

20. P. M. Lebedev, I. N. Onishchenko, Yu. V. Tkach, Ya. B. Fainberg, and V. I. Shevchenko, Sov. J. Plasma Phys. 2, 222 (1976).

21. E. V. Mishin and Ju. Ja. Ruzhin, Adv. Space Res. 1, 47 (1981). https://doi.org/10.1016/0273-1177(81)90271-4

22. E. V. Mishin, Yu. Ya. Ruzhin, and V. A. Telegin, Interaction of Electron Flows with Ionospheric Plasma (Gidrometeoizdat, Leningrad, 1989) [in Russian].

23. V. I. Sotnikov, Yu. A. Omelchenko, V. D. Shapiro, V. I. Shevchenko, M. Ashour-Abdalla, and D. Schriver, Phys Fluids 4, 3562 (1992). https://doi.org/10.1063/1.860363

24. H. L. Rowland, C. L. Chang, and K. Papadopoulos, J. Geophys. Res.: Space Phys. 86, 9215 (1981). https://doi.org/10.1029/JA086iA11p09215 
25. V. P. Tarakanov and E. G. Shustin, Plasma Phys. Rep. 33, 130 (2007). https://doi.org/10.1134/S1063780X07020067

26. N. V. Isaev and E. G. Shustin, Plasma Phys. Rep. 33, 38 42 (2007).

27. I. L. Klykov, V. P. Tarakanov, and E. G. Shustin, Plasma Phys. Rep. 38, 263 (2012). https://doi.org/10.1134/S1063780X12020067

28. L. Yu. Kochmarev, S. B. Lyakhov, A. D. Maiorov, G. G. Managadze, A. I. Chmil', and E. G. Shustin, Sov. J. Plasma Phys. 11, 364 (1985).

29. D. B. Zolotukhin, M. I. Lomaev, E. M. Oks, A. V. Tyunkov, and Yu. G. Yushkov, Plasma Sources Sci. Technol. 28, 035018 (2019). https://doi.org/10.1088/1361-6595/ab0942

30. A. A. Ivanov, Physics of Highly Nonequilibrium Plasmas (Atomizdat, Moscow, 1977) [in Russian].

31. V. M. Atamanov, A. I. Zhuzhunashvili, S. I. Krasheninnikov, and V. V. Shapkin, Sov. J. Plasma Phys. 5, 118 (1979).

32. P. S. Strelkov, Phys.-Usp. 62, 465 (2019). https://doi.org/10.3367/UFNe.2018.09.038443

33. W. N. Hess, Science 164, 1512 (1969). https://doi.org/10.1126/science.164.3887.1512

34. F. Cambou, V. N. Dokukin, V. N. Ivchenko, G. G. Managadze, V. V. Migulin, O. K. Nazarenko, A. T. Nesmyanovich, A. Kh. Pyatsi, R. Z. Sagdeev, and I. A. Zhulin, Space Res. 15, 491 (1975).

35. F. Cambou, J. Lavergnat, V. V. Migulin, A. I. Morozov, B. E. Paton, R. Pellat, A. Kh. Pyatsi, H. Rème, R. Z. Sagdeev, W. R. Sheldon, and I. A. Zhulin, Nature 271 (5647), 723 (1978).

https://doi.org/10.1038/271723a0

36. F. Cambou, V. Dokoukine, J. Lavergnat, R. Pellat, H. Rème, A. Saint-Marc, R. Z. Sagdeev, and I. A. Zhulin, Ann. Geophys. 36, 271 (1980).

37. E. Mishin, V. Ivchenko, and G. Milinevsky, Adv. Space Res. 1, 163 (1981).

https://doi.org/10.1016/0273-1177(81)90286-6

38. B. N. Maehlum, K. Måseide, K. Aarsnes, A. Egeland, B. Grandal, J. Holtet, T. A. Jacobsen, N. C. Maynard, F. Søraas, J. Stadsnes, E. V. Thrane, and J. Trøim, Planet. Space Sci. 28, 259 (1980). https://doi.org/10.1016/0032-0633(80)90017-3

39. B. N. Maehlum, B. Grandal, T. A. Jacobsen, and J. Trøim, Planet. Space Sci. 28, 279 (1980). https://doi.org/10.1016/0032-0633(80)90018-5

40. E. V. Mishin, Yu. Ya. Ruzhin, and V. A. Telegin, Interaction of Electron Flows with Ionospheric Plasma (Gidrometeoizdat, Leningrad, 1989) [in Russian].

41. Yu. Omelchenko, V. Sotnikov, V. Shapiro, and V. Shevchenko, Planet. Space Sci. 40, 535 (1992). https://doi.org/10.1016/0032-0633(92)90172-K

42. N. V. Baranets, Yu. P. Sobolev, M. Ciobanu, J. Vojta, J. Smilauer, Z. Klos, H. Rothkaehl, A. Kiraga, K. Kudela, J. Matišin, V. V. Afonin, B. S. Ryabov, and N. V. Isaev, Plasma Phys. Rep. 33, 995 (2007). https://doi.org/10.1134/S1063780X07120057

43. https://www.andoyaspace.no/2019/11/27/the-ici-5sounding-rocket-launched-074304-ut-nov-26th- from-ny-alesund-svalbard-into-a-beautiful-red-auroral-arc.

44. Artificial Aurora Borealis. https://www.youtube.com/watch?v=SQ3sMTtYP6Y.

45. Yu. S. Korolyev, T. A. Novskova, V. P. Popovich, I. F. Kharchenko, and E. G. Shustin, Kosm. Issled. 13, 730 (1975).

46. W. Bernstein, H. Leinbach, P. Kellogg, S. J. Monson, T. Hallinan, O. K. Garriott, A. Konradi, J. McCoy, P. Daly, B. Baker, and H. R. Anderson, Geophys. Res. Lett. 5, 127 (1978).

https://doi.org/10.1029/GL005i002p00127

47. W. Bernstein, H. Leinbagh, P. Kellogg, S. J. Monson, and T. Hallinan, J. Geophys. Res.: Space Phys. 84, 7271 (1979).

https://doi.org/10.1029/JA084iA12p07271

48. Artificial Particle Beams in Space Plasma Studies (NATO Science Series B, Vol. 79), Ed. by B. Grandal (Plenum, New York, 1982).

49. Active Experiments in Space, ESA Report SP-195 (European Space Agency, Paris, 1983).

50. S. B. Lyachov, A. D. Mayorov, G. G. Managadze, O. A. Povayaev, A. I. Chmil, L. Y. Kochmarev, E. G. Shustin, M. Friedrich, W. Riedler, A. N. Laliashvili, A. Kiraga, Z. Klos, and Z. Kravchic, Planet. Space Sci. 30, 347 (1982). https://doi.org/10.1016/0032-0633(82)90040-X

51. G. G. Managadze, V. M. Balebanov, A. A. Burchudladze, T. I. Gagua, N. A. Leonov, S. B. Lyakhov, A. A. Martinson, A. D. Mayorov, W. K. Riedler, M. F. Friedrich, K. M. Torkar, A. N. Laliashvili, Z. Klos, and Z. Zbyszynski, Planet. Space Sci. 36, 399 (1988). https://doi.org/10.1016/0032-0633(88)90128-6

52. A. Yu. Bessarabskii and E. G. Shustin, Kosm. Issled. 26, 953 (1988).

53. E. V. Mishin, Front. Astron. Space Sci. 6, 14 (2019). https://doi.org/10.3389/fspas.2019.00014

54. J. Ishikava and T. Takagi, J. Appl. Phys. 54, 2911 (1983). https://doi.org/10.1063/1.332493

55. K. D. Schatz and D. N. Ruzic, Plasma Sources Sci. Technol. 2, 100 (1993).

56. R. F. Fernsler, W. M. Manheimer, R. A. Meger, J. Mathew, D. P. Murphy, R. E. Pechacek, and J. A. Gregor, Phys. Plasmas 5, 2137 (1998). https://doi.org/10.1063/1.872886

57. D. Leonhardt, S. G. Walton, and R. F. Fernsler, Phys. Plasmas 14, 057103 (2007). https://doi.org/10.1063/1.2712424

58. S. G. Walton, D. R. Boris, S. C. Hernández, E. H. Lock, Tz. B. Petrova, G. M. Petrov, and R. F. Fernsler, ECS J. Solid State Sci. Technol. 4, N5033 (2015). https://doi.org/10.1149/2.0071506jss

59. D. R. Boris, V. D. Wheeler, N. Nepal, S. B. Qadri, S. G. Walton, and C. R. Eddy, J. Vac. Sci. Technol., A 38, 040801 (2020). https://doi.org/10.1116/6.0000145

60. A. S. Klimov, M. I. Lomaev, E. M. Oks, and A. P. Andreichik, Tech. Phys. 62, 218 (2017). https://doi.org/10.1134/S1063784217020128 
61. A. Klimov and A. Zenin, MATEC Web Conf. 143, 03008 (2018).

https://doi.org/10.1051/matecconf/201814303008

62. A. V. Kazakov, A. V. Medovnik, E. M. Oks, and N. A. Panchenko, J. Phys.: Conf. Ser. 1611, 012014 (2020).

https://doi.org/10.1088/1742-6596/1611/1/012014

63. D. B. Zolotukhin, A. S. Klimov, E. M. Oks, A. V. Tyunkov, Yu. G. Yushkov, and A. A. Zenin, Vacuum 184, 109944 (2021). https://doi.org/10.1016/j.vacuum.2020.109944

64. A. S. Klimov, I. Yu. Bakeev, E. M. Oks, and A. A. Zenin, Ceram. Int. 46, 22276 (2020). https://doi.org/10.1016/j.ceramint.2020.05.306

65. N. V. Isaev, A. I. Chmil', and E. G. Shustin, Plasma Phys. Rep. 30, 263 (2004). https://doi.org/10.1134/1.1687029

66. E. G. Shustin, J. Phys.: Conf. Ser. 1393, 012058 (2019). https://doi.org/10.1088/1742-6596/1393/1/012058

67. N. V. Isaev, M. P. Temiryazeva, Y. V. Fedorov, and E. G. Shustin, Vacuum 83, 1350 (2009). https://doi.org/10.1016/j.vacuum.2009.03.033

68. N. V. Isaev, I. L. Klykov, V. V. Peskov, and E. G. Shustin, Vacuum 85, 711 (2011). https://doi.org/10.1016/j.vacuum.2010.11.004

69. N. V. Isaev, I. L. Klykov, V. V. Peskov, E. G. Shustin, I. V. Vizgalov, and V. A. Kurnaev, Instrum. Exp. Tech.
57, 82 (2014). https://doi.org/10.1134/S002044121401014X

70. Yu. I. Latyshev, A. P. Orlov, V. V. Peskov, E. G. Shustin, A. A. Schekin, and V. A. Bykov, Dokl. Phys, 57, 1 (2012). https://doi.org/10.1134/S1028335812010077

71. E. G. Shustin, N. V. Isaev, I. L. Klykov, V. V. Peskov, V. I. Polyakov, A. I. Rukovishnikov, and M. P. Temiryazeva, Tech. Phys. 58, 245 (2013).

https://doi.org/10.1134/S1063784213020229

72. V. P. Tarakanov, User's Manual for Code KARAT (Berkley Research Associates, Springfield, VA, 1992).

73. V. P. Tarakanov, in Mathematical Modeling: Problems and Results, Ed. by O. M. Belotserkovskii (Nauka, Moscow, 2003), p. 456 [in Russian].

74. V. P. Tarakanov and E. G. Shustin, Vacuum 113, 59 (2015). https://doi.org/10.1016/j.vacuum.2014.12.014

75. E. G. Shustin, V. P. Tarakanov, and K. Ronald, Vacuum 135, 1 (2017). https://doi.org/10.1016/j.vacuum.2016.10.002

76. M. A. Lieberman and A. J. Lichtenberg, Principles of Plasma Discharges and Materials Processing (Wiley, New York, 1994).

77. V. P. Tarakanov, E. G. Shustin, and K. Ronald, Vacuum 165, 262 (2019).

https://doi.org/10.1016/j.vacuum.2019.04.021 